\title{
EMBEDDINGS INTO SIMPLE FREE PRODUCTS
}

\author{
DAVID MEIER
}

\begin{abstract}
We prove that a countable group $G$ can be embedded into a two-generator simple group $S$ which is an amalgamated free product of groups $G * F_{1}$ and $F$, where $F$ and $F_{1}$ are free groups on two generators. $S$ is also the product of two commuting free subgroups. If $G$ has solvable word problem, then we can construct a recursive presentation for $S$.
\end{abstract}

1. Introduction. It is well known that every countable group is a subgroup of a simple two-generator group (see [3] and [7]). The main result of our paper is

THEOREM A. (a) A countable group $G$ can be embedded into a simple two-generator group $S$ which is an amalgamated free product of $G * F_{1}$ and $F$, where $F$ and $F_{1}$ are both free groups on two generators. $S$ is also the product of two commuting free subgroups.

(b) If $G$ has solvable word problem, we can construct the group $S$ of (a) with a recursive presentation.

It was first shown by R. J. Thompson [8] by a construction completely different from ours that a countable group with solvable word problem is a subgroup of a recursively presented finitely generated simple group. Our construction of the group $S$ is similar to the construction of the simple free products of R. Camm [1], or see [6] for a slightly simplified version.

2. The construction of the group $S$. Let $H$ be a countable infinite group with an element $x$ of order at least 5, and assume that the elements of $H$ are numbered by the integers, $H=\left\{g_{i}, i \in \mathbf{Z}\right\}$, such that $g_{0}=1, g_{1}=x, g_{2}=x^{2}, g_{3}=x^{3}, g_{4}=x^{4}$. Let $A=\langle a\rangle$ and $F=\langle b, c\rangle$ be free groups on $a$ and $b, c$, respectively. Then the subgroup $U$ of $A * H$ generated by the elements of the form

$$
u_{i}=a^{i} g_{\rho(i)}, \quad i \in \mathbf{Z}-\{0\},
$$

where $\rho$ is a permutation of $\mathbf{Z}-\{0\}$, is a free group freely generated by the elements $u_{i}, i \in \mathbf{Z}-\{0\}$, with transversal $A$ in $A * H$. Similarly, the elements

$$
v_{j}=b^{j} c^{\sigma(j)}, \quad j \in \mathbf{Z}-\{0\},
$$

of $F$, where $\boldsymbol{\sigma}$ is a second permutation of $\mathbf{Z}-\{0\}$, freely generate a free subgroup $V$ of $F$ with transversal $B=\langle b\rangle$.

Received by the editors April 10, 1983.

1980 Mathematics Subject Classification. Primary 20E06, 20E32, 20F10. 
We can therefore form the amalgamated free product

$$
S=\left\langle A * H * F ; u_{i}=v_{\tau(i)}, i \in \mathbf{Z}-\{0\}\right\rangle,
$$

$\tau$ is a third permutation of $\mathbf{Z}-\{0\}$.

By the normal form theorem for amalgamated free products (see Theorem 4.4 in [5]), $S=U\langle a, b\rangle$, where $\langle a, b\rangle$ is free on $a$ and $b . S$ is therefore the product of two commuting free subgroups.

We now choose $\rho, \sigma, \tau$ in such a way that $S$ is a simple two-generator group.

3. The permutations $\rho, \sigma, \tau$. For $1 \leqslant i \leqslant 4, \rho, \sigma, \tau$ are defined by the equations

$$
\begin{aligned}
a g_{1} & =b c, \\
a^{2} g_{2} & =b^{2} c^{3}, \\
a^{3} g_{3} & =b^{4} c^{4}, \\
a^{4} g_{4} & =b^{3} c^{2} .
\end{aligned}
$$

For $-4 \leqslant i \leqslant-1$ we set $a^{i} g_{i}=b^{i} c^{i}$. Assume now that $z_{i}=a^{i_{1}} b^{j_{1}} \cdots b^{j_{n}} a^{i_{n+1}}, i=$ $0,1,2, \ldots$, is a recursive enumeration of the elements of $\langle a, b\rangle, z_{0}=$ identity element. For $|i|>4$ the permutations are inductively defined by going through the list of elements $z_{i}$, beginning with $z_{1}$, in the following way. Assume that $n_{0}$ is the smallest positive number for which $\rho, \sigma, \tau$ are not yet defined and $z_{i}=a^{i_{1}} b^{j_{1}} \ldots$ $a^{i_{n}} b^{j_{n}} a^{i_{n+1}}$ is the next word on our list. Note that $i_{1}=0$ or $i_{n+1}=0$ are allowed. Then set

$$
x_{i}=a^{i_{1}} g_{j_{1}} \cdots a^{i_{n}} g_{j_{n}} a^{i_{n+1}}, \quad w_{i}=b^{i_{1}} c^{j_{1}} \cdots b^{i_{n}} c^{j_{n}} b^{i_{n+1}} .
$$

If $z_{i}=a^{i_{1}}$, if $i_{1}=-i_{n+1}$, or if $i_{1}=-i_{n+1}-1$, proceed to (III), otherwise:

(I)(a) Choose distinct numbers $p_{i}, i=1, \ldots, 2 n$, all $p_{i} \geqslant n_{0}$, such that $p_{2 n}=p_{0}-$ $i_{n+1}$, where $p_{0}$ is defined by $p_{1}=p_{0}+i_{1}$, and such that $p_{2 k-1}-p_{2 k-2}=i_{k}$ for $k=2, \ldots, n$.

(I)(b) Choose distinct numbers $\pi_{i}, i=1, \ldots, 2 n,\left|\pi_{i}\right| \geqslant n_{0}$ for all $i$, such that $g_{\pi_{2 k-1}} g_{\pi_{2 k}}^{-1}=g_{j_{k}}$ for $k=1, \ldots, n$.

(I)(c) Choose distinct numbers $q_{i}, i=1, \ldots, 2 n, q_{i} \geqslant n_{0}$ such that $q_{2 k-1}-q_{2 k}=j_{k}$ for $k=1, \ldots, n$.

Then: set $n_{1}=\max \left(p_{i},\left|\pi_{i}\right|, q_{i}, i=1, \ldots, 2 n\right)$ and $\rho\left(p_{i}\right)=\pi_{i}, \sigma\left(p_{i}\right)=q_{i}$ for $i=$ $1, \ldots, 2 n$; complete $\rho$ and $\sigma$ to permutations of $I_{1}=\left[-n_{1},-n_{0}\right] \cup\left[n_{0}, n_{1}\right]$; and set $\tau(i)=i$ for all $i$ in $I_{1}$.

(II)(a) Choose distinct numbers $r_{i}, i=1, \ldots, 2 n, r_{i}>n_{1}$ for all $i$, such that $r_{2 n}=r_{0}-i_{n+1}-1$, where $r_{0}$ is defined by $r_{1}=r_{0}+i_{1}$, and such that $r_{2 k-1}-r_{2 k-2}$ $=i_{k}$ for $k=2, \ldots, n$.

(II)(b) Choose distinct numbers $\rho_{i}, i=1, \ldots, 2 n,\left|\rho_{i}\right|>n_{1}$ for all $i$, such that $g_{\rho_{2 k-1}} g_{\rho_{2 k}}^{-1}=g_{j_{k}}$ for $k=1, \ldots, n$.

(II)(c) Choose distinct numbers $\sigma_{i}, i=1, \ldots, 2 n, \sigma_{i}>n_{1}$ for all $i$, such that $\sigma_{2 k-1}-\sigma_{2 \mathrm{k}}=j_{k}$ for $k=1, \ldots, n$.

Then: set $n_{2}=\max \left(r_{i},\left|\rho_{i}\right|, \sigma_{i}, i=1, \ldots, 2 n\right)$ and $\rho\left(r_{i}\right)=\rho_{i}, \sigma\left(r_{i}\right)=\sigma_{i}$ for $i=$ $1, \ldots, 2 n$; complete $\rho$ and $\sigma$ to permutations of $I_{2}=\left[-n_{2},-n_{1}-1\right] \cup\left[n_{1}+1, n_{2}\right]$; and set $\tau(i)=i$ for all $i$ in $I_{2}$. 
(III)(a) Choose numbers $s_{i}, i=0, \ldots, 2 n+1, s_{i}>n_{2}$ for all $i$,

$$
s_{i} \text { distinct for } \begin{cases}i=1, \ldots, 2 n & \text { if } i_{1}=0=i_{n+1}, \\ i=0, \ldots, 2 n & \text { if } i_{1} \neq 0=i_{n+1}, \\ i=1, \ldots, 2 n+1 & \text { if } i_{1}=0 \neq i_{n+1}, \\ i=0, \ldots, 2 n+1 & \text { if } i_{1} \neq 0 \neq i_{n+1},\end{cases}
$$

such that $s_{2 k-1}-s_{2 k-2}=-i_{k}$ for $k=1, \ldots, n+1$. Note that if $i_{1}=0$, then $s_{0}=s_{1}$; and if $i_{n+1}=0$, then $s_{2 n}=s_{2 n+1}$.

(III)(b) Choose distinct numbers $\nu_{i}, i=1, \ldots, 2 n, \nu_{i}>n_{2}$ for all $i$, such that $\nu_{2 k}-\nu_{2 k-1}=-j_{k}$ for $k=1, \ldots, n$.

Then: set $n_{3}=\max \left(s_{i}, \nu_{j}, i=0, \ldots, 2 n+1, j=1, \ldots, 2 n\right)$ and $\tau\left(s_{i}\right)=\nu_{i}$ for $i=1, \ldots, 2 n$; complete $\tau$ to a permutation of $I_{3}=\left[-n_{3},-n_{2}-1\right] \cup\left[n_{2}+1, n_{3}\right]$; and set $\sigma(i)=\rho(i)=i$ for $i \in I_{3}$.

(III)(c) Choose numbers $\rho_{i}, i=0, \ldots, 2 n+1,\left|\rho_{i}\right|>n_{3}$ for all $i, \rho_{i}$ distinct whenever the $s_{i}$ in (III)(a) are distinct, such that $g_{\rho_{2 k}}^{-1} g_{\rho_{2 k+1}}=g_{s_{2 k}}^{-1} g_{s_{2 k+1}}$ for $k=$ $0, \ldots, n$.

Note that $s_{0}=s_{1}$ implies $\rho_{0}=\rho_{1}$ and $s_{2 n}=s_{2 n+1}$ implies $\rho_{2 n}=\rho_{2 n+1}$. Then: set $t_{i}=s_{i}+n_{3}-n_{2}$ for $i=0, \ldots, 2 n+1 ; \mu_{i}=\nu_{i}+n_{3}-n_{2}$ for $i=1, \ldots, 2 n ; n_{4}=$ $\max \left(t_{i}, \mu_{j},\left|\rho_{i}\right|, \quad i=0, \ldots, 2 n+1, j=1, \ldots, 2 n\right) ; \tau\left(t_{i}\right)=\mu_{i}, \sigma\left(\mu_{i}\right)=\mu_{i}$ for $i=$ $1, \ldots, 2 n, \rho\left(t_{i}\right)=\rho_{i}$ for $i=0, \ldots, 2 n+1$; and complete $\tau, \sigma, \rho$ to permutations of $I_{4}=\left[-n_{4},-n_{3}-1\right] \cup\left[n_{3}+1, n_{4}\right]$.

Now proceed to word $z_{i+1}$.

Note that integers $p_{i}, \pi_{i}, q_{i}, r_{j}, \rho_{j}, \sigma_{j}, s_{k}, \nu_{k}, \rho_{k}$ satisfying the conditions of (I), (II), and (III), respectively, always exist, since at any stage of the construction we can choose from all but finitely many integers.

The case $z_{i}=a^{i_{1}}$ fits into the above construction with $n=0$ if we omit the $\nu$ and $\mu$ part in (III)(b) and (c).

Properties of $\rho, \sigma, \tau$. (i) If $x_{i}=a^{i_{1}} g_{j_{1}} \cdots a^{i_{n}} g_{j_{n}} a^{i_{n+1}}$ and $w_{i}=b^{i_{1}} c^{j_{1}} \cdots b^{i_{n}} c^{j_{n}} b^{i_{n+1}}$ such that $x_{i} \neq a^{i_{1}}$ and $-i_{n+1} \neq i_{1} \neq-i_{n+1}-1$, then, in the notation of (I) and (II),

(a1) $a^{p_{0}} x_{i} a^{-p_{0}}=u_{p_{1}} u_{p_{2}}^{-1} \cdots u_{p_{2 n-1}} u_{p_{2 n}}^{-1}$

(a2) $b^{p_{0}} w_{i} b^{-p_{0}}=v_{p_{1}} v_{p_{2}}^{-1} \cdots v_{p_{2 n-1}} v_{p_{2 n}}^{-1}$ and $u_{p_{i}}=v_{p_{i}}$ for $i=1, \ldots, 2 n$;

(b1) $a^{r_{0}} x_{i} a^{-r_{0}}=u_{r_{1}} u_{r_{2}}^{-1} \cdots u_{r_{2 n-1}} u_{r_{2 n}}^{-1} a^{-1}$;

(b2) $b^{r_{0}} w_{i} b^{-r_{0}}=v_{r_{1}} v_{r_{2}}^{-1} \cdots v_{r_{2 n-1}} v_{r_{2 n}}^{-1} b^{-1}$ and $u_{r_{i}}=v_{r_{i}}$ for $i=1, \ldots, 2 n$.

Proof. We show (a1) and use the definitions of $\rho, \sigma, \tau$ in (I); (a2), (b1), and (b2) are similar.

$$
\begin{aligned}
& \begin{aligned}
a^{p_{0}} x_{i} a^{-p_{0}} & =\underbrace{a^{p_{0}} a^{i_{1}}} \overbrace{g_{\pi_{1}} g_{\pi_{2}}^{-1}}^{g_{j_{1}}} \overbrace{a^{-p_{2}} a^{p_{3}}}^{a^{i_{2}}} \ldots \overbrace{a^{-p_{2 n-2} a^{p_{2 n-1}}}}^{a_{g_{\pi_{2 n-1}} g_{\pi_{2 n}}^{-1}}^{a^{i_{n}}}} \underbrace{g_{j_{n}}}_{a^{-p_{2 n}}} \underbrace{}_{u^{i_{n+1}} a^{-p_{0}}}
\end{aligned} \\
& =u_{p_{1}} \quad u_{p_{2}}^{-1} \quad u_{p_{3}} \cdots u_{p_{2 n-2}}^{-1} \quad u_{p_{2 n-1}} \quad u_{p_{2 n}}^{-1} \text {. }
\end{aligned}
$$

(ii) If $z_{i}=a^{i_{1}} b^{j_{1}} \cdots a^{i_{n}} b^{j_{n}} a^{i_{n+1}}$, then, in the notation of $\S 2$,

(c) $z_{i}^{-1} u_{s_{0}} u_{t_{0}}^{-1} z_{i}=u_{s_{2 n+1}} u_{t_{2 n+1}}^{-1}$, with $s_{0} \neq s_{2 n+1}$. 
Proof. We use the notation of $\S 2$, (III).

$$
\begin{aligned}
& z_{i}^{-1} u_{s_{0}} u_{t_{0}}^{-1} z_{i} \\
& =\cdots a^{-1_{2}} b^{-j_{1}} a^{-i_{1}} a^{s_{0}} g_{s_{0}} g_{\rho_{0}}^{-1} a^{-t_{0}} a^{i_{1}} b^{j_{1}} a^{i_{2}} \ldots \\
& =\cdots a^{-i_{2}} b^{-j_{1}} a^{s_{0}-i_{1}} g_{s_{0}} g_{\rho_{0}}^{-1} a^{-\left(t_{0}-t_{i}\right)} b^{j_{1}} a^{i_{2}} \cdots \\
& =\cdots a^{-i_{2}} b^{-j_{1}} a^{s_{1}} g_{s_{1}} \underbrace{\left(g_{\rho_{0}} g_{s_{0}}^{-1} g_{s_{1}}\right)^{-1}} a^{-t_{1}} b^{j_{1}} a^{i_{2}} \cdots \\
& =\cdots a^{-i_{2}} b^{-j_{1}} a^{s_{1}} g_{s_{1}} g_{\rho_{1}}^{-1} a^{-t_{1}} b^{j_{1}} a^{i_{2}} \ldots \\
& \text { (now use the fact that } \tau\left(s_{i}\right)=\nu_{i}, \tau\left(t_{i}\right)=\mu_{i} \text { ) } \\
& =\cdots a^{-i_{2}} b^{-j_{1}} b^{\nu_{1}} c^{\nu_{1}} c^{-\mu_{1}} b^{-\mu_{1}} b^{j_{1}} a^{i_{2}} \ldots \\
& =\cdots a^{-i_{2}} b^{-j_{1}+\nu_{1}} c^{-j_{1}+\nu_{1}} c^{-\left(-j_{1}+\mu_{1}\right)} b^{-\left(-j_{1}+\mu_{1}\right)} a^{i_{2}} \ldots \\
& =\cdots a^{-i_{2}} b^{\nu_{2}} c^{\nu_{2}} c^{-\mu_{2}} b^{-\mu_{2}} a^{i_{2}} \ldots \\
& =\cdots a^{-i_{2}} a^{s_{2}} g_{s_{2}} g_{\rho_{2}}^{-1} a^{-t_{2}} a^{i_{2}} \ldots \\
& =\cdots a^{-i_{2}} u_{s_{2}} U_{t_{2}}^{-1} a^{i_{2}} \ldots
\end{aligned}
$$

By proceeding in the same manner we finally obtain

$$
=u_{s_{2 n+1}} u_{t_{2 n+1}}^{-1} \text {. }
$$

The choice of the $s_{i}$ in (III)(a) guarantees that $s_{0} \neq s_{2 n+1}$ for any $z_{i} \neq 1$.

4. The group $S$ is generated by two elements. Since for every element $g_{i}$ in $H$, there is a relation of the form $a^{r} g_{i}=b^{s} c^{t}, S$ is obviously generated by $a, b$, and $c$. If we rewrite (1)-(4) by using the fact that $g_{1}=x, \ldots, g_{4}=x^{4}$, we get

(1) $a x=b c$,

(2) $a^{2} x^{2}=b^{2} c^{3}$

(3) $a^{3} x^{3}=b^{4} c^{4}$,

(4) $a^{4} x^{4}=b^{3} c^{2}$.

It is shown in [1, p. 76] that (1) and (2) can be used to express $a, b$, and $c$ by $b^{-1} a$ and $b c$.

5. The group $S$ is simple. The strategy of the proof is as follows. We first show that $\langle S ; a=b\rangle$ is trivial. Then we show that for any nontrivial element $x$ in $U$, the additional relation $x=1$ implies $a=b$. Finally, we show that for any nontrivial element $s$ of $S$, the additional relation $s=1$ implies a nontrivial relation $x=1$ with $x$ in $U$.

(i) $\langle S ; a=b\rangle$ is trivial. Since $S$ is generated by $a, b$, and $c$ by $\S 4$, it is enough to show that $a=b$ implies $a=b=c=1$. This follows easily from relations (1)-(4).

(ii) For any nontrivial $x_{i}=a^{i_{1}} g_{j_{1}} a^{i_{2}} \cdots g_{j_{n}} a^{i_{n+1}}$ in $U, x_{i}=1$ implies $a=b$. Since $x_{i} \in U, x_{i}=u_{k_{1}}^{ \pm 1} u_{k_{2}}^{ \pm 1} \cdots u_{k_{r}}^{ \pm 1}$. If we replace the relator $x_{i}$ by a cyclically reduced conjugate in the $u_{i}$, and then by its inverse if necessary, we can assume that $x_{i} \neq a^{i_{\mathrm{i}}}$ and $-i_{n+1} \neq i_{1} \neq-i_{n+1}-1$. As in $\S 2$ we set $w_{i}=b^{i_{1}} c^{j_{1}} b^{i_{2}} \cdots c^{j_{n}} b^{i_{n+1}}$. We show next that the relator $x_{i}$ implies a relator $x_{j}$ with the property $x_{j}=w_{j}$. We use the 
notation of the previous sections and claim that $x_{j}=a^{p_{0}} x_{i} a^{-p_{0}}$ has the desired properties. By (a1) in $\S 3$,

$$
x_{j}=u_{p_{1}} u_{p_{2}}^{-1} \cdots u_{p_{2 n-1}} u_{p_{2 n}}^{-1}=v_{p_{1}} v_{p_{2}}^{-1} \cdots v_{p_{2 n-1}} v_{p_{2 n}}^{-1} .
$$

Now use the definitions of $\rho, \sigma, \tau$ in (I) to rewrite $x_{j}$ in $A * H$ and in $F$; then

$$
\begin{aligned}
x_{j} & =a^{p_{1}} g_{j_{1}} a^{i_{2}} \cdots g_{j_{n}} a^{-p_{2 n}} \quad \text { in } A * H \\
& =b^{p_{1}} c^{j_{1}} b^{i_{2}} \cdots c^{j_{n}} b^{-p_{2 n}} \quad \text { in } F ;
\end{aligned}
$$

therefore $x_{j}=w_{j}$. Note also that, since $p_{1}=p_{0}+i_{1}$ and $p_{2 n}=p_{0}-i_{n+1}$, the condition $i_{1} \neq-i_{n+1}-1$ for $x_{i}$ implies $p_{1} \neq p_{2 n}-1$ for $x_{j}$.

Now we use (II) of $\S 2$ and (b1) and (b2) in $\S 3$ for $x_{j}$ and $w_{j}$ to get

$$
\begin{aligned}
& a^{r_{0}} x_{j} a^{-r_{0}}=u_{r_{1}} u_{r_{2}}^{-1} \cdots u_{r_{2 n}}^{-1} a^{-1}, \\
& b^{r_{0}} w_{j} b^{-r_{0}}=v_{r_{1}} v_{r_{2}}^{-1} \cdots v_{r_{2 n}}^{-1} b^{-1},
\end{aligned}
$$

and

$$
u_{r_{i}}=v_{r_{i}} \text { for } i=1, \ldots, 2 n \text {. }
$$

Therefore, $b a^{-1}$, the product of the relators $b^{r_{0}} w_{j}^{-1} b^{-r_{0}}$ and $a^{r_{0}} x_{j} a^{-r_{0}}$, is a relator of $\left\langle S ; x_{i}=1\right\rangle$.

(iii) If $s$ is a nontrivial element of $S$, then the additional relation $s=1$ implies a nontrivial relation $x=1$ for some $x$ in $U$.

Since $S=U\langle a, b\rangle$, the element $s$ is a product $x z_{i}$ with $x$ in $U$ and $z_{i}=a^{i_{1}} b^{j_{1}} \ldots$ $a^{i_{n}} b^{j_{n}} a^{i_{n+1}}$ with possibly $i_{1}=0$ or $i_{n+1}=0$. The assertion is trivial if $z_{i}=1$ : we therefore assume $z_{i} \neq 1$. By property (c) in $\S 3$

$$
z_{i}^{-1} u_{s_{0}} u_{t_{0}}^{-1} z_{i}=u_{s_{2 n+1}} u_{t_{2 n+1}}^{-1} \text { with } s_{0} \neq s_{2 n+1} \text { and } t_{0} \neq t_{2 n+1} .
$$

The relation $s=1$ implies $z_{i}^{-1}=x$; therefore

$$
x u_{s_{0}} u_{t_{0}}^{-1} x^{-1}=u_{s_{2 n+1}} u_{t_{2 n+1}}^{-1} .
$$

The elements $\ldots, u_{0}, \ldots, u_{s_{0}}, \ldots, u_{s_{2 n+1}}, \ldots, u_{t_{0}}, \ldots, u_{t_{2 n+1}}, \ldots$ are free generators of the subgroup $U$ in $G$. Therefore the set

$$
\ldots, u_{0}, \ldots, u_{s_{0}} u_{t_{0}}^{-1}, \ldots, u_{s_{2 n+1}} u_{t_{2 n+1}}^{-1}, \ldots, u_{t_{0}}, \ldots, u_{t_{2 n+1}}, \ldots,
$$

is also a free generating set of $U$. Since distinct free generators in a free group are not conjugate, $(*)$ is a nontrivial relation in $U$.

6. Proof of Theorem A. If $G$ is a countable group, then $H=G *\langle x\rangle$, where $\langle x\rangle$ is infinite cyclic, has the properties required for the construction of the group $S$. Now write $F_{1}$ for the free group $\langle x, a\rangle$; then the proof of (a) is complete.

If $G$ has solvable word problem, and $G=\langle T ; R\rangle$ is a presentation of $G$ with respect to which the word problem is solvable, then the word problem of $H=$ $\langle T, x ; R\rangle$ is solvable with respect to the given presentation, and we can therefore effectively enumerate the elements of $H$.

The permutations $\rho, \sigma, \tau$ are (almost) defined by an algorithm; thus, once we are able to effectively enumerate the elements of $H$, we can effectively produce the 
relations $a^{i} g_{\rho(i)}=b^{\tau(i)} c^{\sigma(\tau(i))}$ of $S$. The relations of the presentation

$$
S=\left\langle T, x, a, b, c ; R, a^{i} g_{\rho(i)}=b^{\tau(i)} c^{\sigma(\tau(i))}, i \in \mathbf{Z}-\{0\}\right\rangle
$$

are therefore recursively enumerable. This proves Theorem $A(b)$. Theorem $A(b)$, together with the well-known result of Kusnetsov [4, p. 191], gives a new proof of the following result of $\mathbf{R}$. J. Thompson [8], which is in turn a sharpened version of a result of W. W. Boone and G. Higman [2].

COROLlaRY B. A finitely generated group $G$ has solvable word problem if and only if it is a subgroup of a recursively presented finitely generated simple group.

We close with three remarks.

1. Since $G$ is a free factor of one of the factors of the amalgamated free product $S$, the embedding $G \rightarrow S$ has the following additional property: If the elements $g_{i}$ and $g_{j}$ of $G$ are not conjugate in $G$, then they are not conjugate in $S$. Use Theorem 4.6 in [5]. It shares this property with the embedding constructed by R. J. Thompson [8].

2: The group $S$ is an amalgamated free product, we have therefore control over the torsion elements of $S$. They are the conjugates of the torsion elements of $G$. Especially, if $G$ is torsionfree, then $S$ is torsionfree.

3. If $G$ is the trivial group, then $H=G *\langle x\rangle$ is infinite cyclic and

$$
S=\left\langle x, a, b, c ; a^{i} x^{\rho(i)}=b^{\tau(i)} c^{\sigma(\tau(i))}\right\rangle .
$$

This is the situation studied in [1] and [6].

\section{REFERENCES}

1. R. Camm, Simple free products, J. London Math. Soc. 28 (1953), 66-76.

2. W. W. Boone and G. Higman, An algebraic characterization of the solvability of the word problem, J. Austral. Math. Soc. 18 (1974), 41-53.

3. A. P. Goryushkin, Imbedding of countable groups in two-generator groups, Mat. Zametki 16 (1974), 231-235.

4. R. C. Lyndon and P. E. Schupp, Combinatorial group theory, Springer-Verlag, Heidelberg, 1977.

5. W. Magnus, A. Karrass and D. Solitar, Combinatorial group theory, Wiley, New York, 1966.

6. D. Meier, $A$ note on simple free products, Houston J. Math. 9 (1983), 501-504.

7. P. E. Schupp, Embeddings into simple groups, J. London Math. Soc. 13 (1976), 90-94.

8. R. J. Thompson, Embeddings into finitely generated simple groups which preserve the word problem, Word Problems. II, North-Holland, Amsterdam, 1980, pp. 401-441.

Department of Mathematics, University of Alberta, Edmonton, Alberta, T6G 2G1, Canada Current address: Pilgerweg 1, 8044 Zürich, Switzerland 